\title{
How to make an efficient propaganda
}

\author{
T. Carletti ${ }^{1}$, D. Fanelli ${ }^{2,3}$, S. Grolli $^{4}$ and A. Guarino ${ }^{4}$ \\ 1 Département des Mathématiques FUNDP \\ rempart de la Vierge 8, B 5000 Namur, Belgium \\ 2 Dipartimento di Energetica, Università di Firenze and INFN \\ via S. Marta, 3, 50139 Firenze, Italy \\ 3 Department of Cell and Molecular Biology, Karolinska Institute \\ SE-17771 Stockholm, Sweden \\ 4 Université de la Polynésie Française, BP 6570 Faa'a, 98702 Tahiti, French Polynesia
}

\begin{abstract}
The effects of propaganda are analyzed in an opinion dynamics model in which, under certain conditions, individuals adjust their opinion as a result of random binary encounters. The aim of this paper is to study under what conditions propaganda changes the opinion dynamics of a social system. Four different scenarios are found, characterized by different sensitivities to the propaganda. For each scenario the maximum efficiency of propaganda is attained following a given strategy that is here outlined.
\end{abstract}

Introduction. - The link between physics and sociology is historically profound. At the end of the XVII century, when G. Galilei and I. Newton gave birth to classical physics, philosophers proposed to apply similar deterministic laws to the broad fields of political and social sciences [1-3]. This idea was further strengthened by the discovery of universal demographic constants involving parameters strongly affected by individual free will or happenstance, such as the number of weddings, crimes and deaths [4]. The philosopher A. Comte, then introduced "social physics", a foundation of modern sociology which studies social dynamics using deterministic laws $[5,6]$. As a typical social system is composed of a high number of individuals, statistics is the key tool for a quantitative study. Opinion dynamics is one of the fields of sociology that has interested physicists the most in the latest years. Opinion dynamics models can be divided into two large classes. The first class is represented by models based on binary opinions [7-11], in which social actors update their opinions as a result of social influence, often according to a kind of majority rule. The other class of opinion dynamics models considers the opinion as a continuous variable [12-17]. In this paper we focus on the latter and investigate the effect of propaganda. Indeed, we consider a model where agents adjust continuous opinions as a result of random binary encounters whenever their difference in opinion is below a given threshold [17]. This model is here termed Continuous Opinions with Threshold (COT) model and it is closely related to the "compromise model" studied in [18]. We are in particular interested in analyzing under what conditions propaganda affects the dynamics of the group's opinion. Here, propaganda is a "message" that touches every individual at the same time.

The paper is organized as follows: first, we introduce the model and define the parameters involved. Then we show the results of the numerical simulations and compare them with dedicated analytical estimates. In conclusion, we will try to relate the mathematical results to actual social systems. 
The model. - In our model, the system is formed by $N$ individuals $i$. Each individual has two characteristics. The first one is its opinion $O_{i}$. The opinion is the individual position on a given subject. This picture could apply to many different fields such as politics, marketing, religion, etc. The opinion $O_{i}$ is represented by a number between 0 and 1 that symbolizes the extremes: left and right in politics, atheism and faith in religion, etc. To each individual $i$ a second parameter $\sigma_{i}$ between 0 and 1 is also associated, which can be interpreted as the openness of mind or uncertainty about their own opinion. In this paper we study the case where all the individuals have the same openness of mind, that is $\sigma_{i}=\sigma, \forall i$.

At each time step $t$, corresponding to sampling a pair of individuals, two random chosen individuals meet: if their difference in opinion is smaller than the threshold $\sigma$, their opinion tends to converge. Otherwise, their opinion remains unaltered. Mathematically

$$
\begin{array}{ll}
\text { if } \quad\left|O_{i}(t)-O_{j}(t)\right|>\sigma \Rightarrow & O_{i}(t+1)=O_{i}(t), \quad O_{j}(t+1)=O_{j}(t), \\
\text { if } \quad\left|O_{i}(t)-O_{j}(t)\right|<\sigma \Rightarrow \quad & O_{i}(t+1)=O_{i}(t)+\mu\left[O_{j}(t)-O_{i}(t)\right], \\
& O_{j}(t+1)=O_{j}(t)+\mu\left[O_{i}(t)-O_{j}(t)\right],
\end{array}
$$

where $\mu$ is the convergence parameter whose value may range from 0 to 1 . It has been shown that $\mu$ only influences the time needed for the system to go to equilibrium [17]. The dynamics and behaviour of the system are not affected by this parameter. In this paper we then use the fixed value $\mu=0.5$, which minimizes this transient. Within this framework, a master equation for the distribution $P(x, t)$, i.e. the fraction of agents that have opinions in the range $[x, x+\mathrm{d} x]$ can be derived. This alternative formulation opens up the perspective of implementing a very efficient numerical integration scheme [18].

The main result of the COT model is that, depending on the value $\sigma$, the process may lead to a consensus among the individuals or to a fragmentation in $n_{c}$ clusters of opinions. It has been found that $n_{c} \approx \operatorname{Integer}\left(\frac{1}{2 \sigma}\right)[17,18]$. The smaller the $\sigma$, the more the population will be divided in small groups sharing a common opinion, while for large $\sigma$ the population will reach a complete consensus with an agreement of all the individuals.

Notice that in the COT model only two individuals at a time can interact. We here propose to enrich this picture by introducing an effective propaganda, modeled as an extended periodic perturbation that interacts instantaneously with the whole population. This perturbation can represent a political propaganda, the advertising of a product, a religious message of an external subject who wants to convince the individuals to adhere to a particular constant opinion $O_{p}$. Therefore, in addition to the previous interactions, all the opinions are now simultaneously confronted with the exterior opinion $\left(O_{p}\right)$ every $T$ individual interactions, $T$ labeling the propaganda period. If the difference between the individual opinion $O_{i}$ and the propaganda opinion $O_{p}$ is smaller than $\sigma$, the individual opinion gets closer to the propaganda. Otherwise it remains unchanged. Mathematically

$$
\begin{aligned}
& \text { if }\left|O_{i}(t)-O_{p}\right|>\sigma \Rightarrow O_{i}(t+1)=O_{i}(t) \text {, } \\
& \text { if }\left|O_{i}(t)-O_{p}\right|<\sigma \Rightarrow O_{i}(t+1)=O_{i}(t)+\mu_{p}\left[O_{p}-O_{i}(t)\right] \text {, }
\end{aligned}
$$

where $\mu_{p}$ is the convergence parameter whose value may range from 0 to 0.5 . In order to quantify the effectiveness of propaganda, we define the propaganda efficiency $\left(E_{p}\right)$ as the percentage of individuals convinced, i.e. that share the same opinion of the propaganda when the equilibrium is reached.

Results. - Before studying the effects of propaganda, let us provide an analytical estimate of the characteristic relaxation time, hereafter termed $T_{c}$, associated to the COT-like dynamics. $T_{c}$ measures the time required for the system to converge to its asymptotic state. 


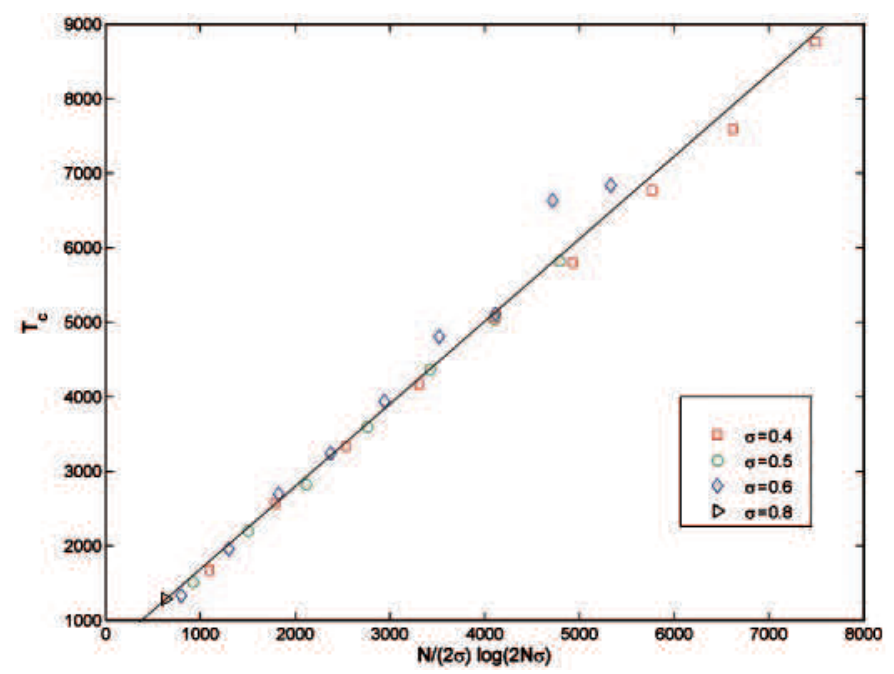

Fig. 1 - The relaxation time $T_{c}$ as a function $\frac{N}{2 \sigma} \ln (2 \sigma N)$. Symbols refer to numerical simulations carried out for different values of $\sigma$, see the legend. As predicted by the proposed theory, a very good linear correlation is observed. The numerical relaxation time is measured by performing the histogram of the opinion distribution at every time step. The convergence is assumed to be reached when all the bins are zero but one. The slope of the continuous curve depends on the chosen binning size.

Consider $N^{\prime}$ opinions uniformly distributed in a finite interval, whose length is smaller than the threshold $\sigma$. At each iteration the number of distinct opinions is reduced by a factor 2 , because of the choice for $\mu$. Hence, one needs approximately $\ln \left(N^{\prime}\right) / \ln (2)$ steps to attain the final convergence state. Moreover, a multiplicative factor $1 / \sigma$ has to be introduced to properly account for the finite range of the interaction, $\sigma$ being the probability that two randomly chosen individuals have opinions closer than $\sigma$. In our case, $n_{c} \approx \operatorname{Integer}\left(\frac{1}{2 \sigma}\right)$ clusters develop, each of them being constituted by $N^{\prime}=2 N \sigma$ elements. Therefore, combining the above ingredients, one ends up with the following estimate for the convergence time $T_{c}$ :

$$
T_{c} \approx \frac{N}{2 \sigma} \frac{\ln (2 \sigma N)}{\ln (2)} .
$$

The functional dependence on $N$ and $\sigma$ is in agreement with the results of numerical simulations, as clearly displayed in fig. 1.

Let us now turn to the COT model with propaganda. The simulations show that the efficiency of the propaganda $E_{p}$ depends on three parameters, the openness of mind $\sigma$, the external opinion $O_{p}$ and propaganda period $T$, while keeping $\mu_{p}$ constant. In the following we shall assume $\mu_{p}=\mu=0.5$ and we observe that the qualitative results we obtain do not depend on such a choice of parameters. In the plane $\left(\sigma, O_{p}\right)$, hereafter named phase diagram, we show that we can define four different phases for the system, fig. 2. Each phase is characterized by a different dependence of the efficiency $E_{p}$ on the propaganda period $T\left({ }^{1}\right)$.

In the range $\sigma \geq\left|O_{p}-\frac{1}{2}\right|+\frac{1}{2}$, zone I in fig. 2, the behaviours are trivial. We always obtain a $100 \%$ efficiency $E_{p}$, no matter what the value of the propaganda opinion $O_{p}$ and period $T$ are. Indeed, in this range $\sigma+O_{p} \geq 1$, that means that propaganda acts directly on every individual.

$\left({ }^{1}\right)$ Notice that for different values of $\mu_{p}$ the system displays qualitatively analogous behaviours even though a different partition of the phase diagram is observed. 


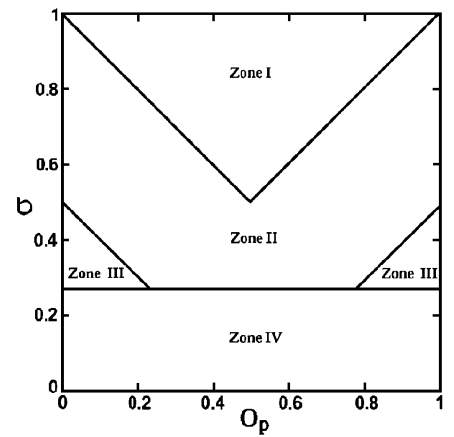

Fig. 2
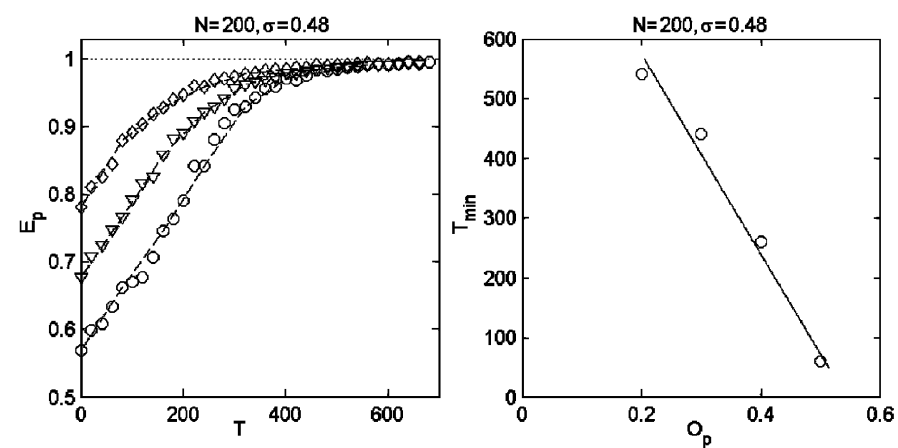

Fig. 3

Fig. 2 - Phase diagram $\left(\sigma, O_{p}\right)$. Four different phase zones can be defined. Each zone is characterized by a different dependence of the efficiency $E_{p}$ on the propaganda period $T$. In zone I, $\sigma>\mid O_{p}-$ $0.5 \mid+0.5, E_{p}$ is always $100 \%$, independently of the value of $T$. In zone II, $\max \left\{0.28,\left|0.5-O_{p}\right|\right\}<$ $\sigma<\left|0.5-O_{p}\right|+0.5 . E_{p}$ is $100 \%$ only if $T>T_{\min }$. Zones III, $0.28<\sigma<0.5-O_{p}$, efficiency $E_{p}$ is $100 \%$ only if $T_{\min }<T<T_{\max }$. Finally, in zone IV, propaganda has only a local effect; it can never reach $100 \%$ efficiency.

Fig. 3 - Analysis of zone II. Left: the efficiency $E_{p}$ as a function of the propaganda period $T$ for different values of $O_{p}$ in the case $N=200$ and $\sigma=0.48$. The open circles (०), the diamonds $(\diamond)$ and the triangles $(\nabla)$ represent, respectively, the case $O_{p}=0.1, O_{p}=0.2$ and $O_{p}=0.3$. Right: the value of $T_{\text {min }}$ as a function of $O_{p}$ in the case $N=200$ and $\sigma=0.48$.

More interesting results are obtained in the range $\max \left(0.28,\left|O_{p}-\frac{1}{2}\right|\right) \leq \sigma \leq\left|O_{p}-\frac{1}{2}\right|+\frac{1}{2}$ of the phase diagram $\left(\sigma, O_{p}\right)$, zone II in fig. 2. Here, propaganda can obtain $100 \%$ efficiency, that is, all the people have a final opinion equal to $O_{p}$. In order to attain the maximum efficiency, the propaganda period $T$ must be larger than a minimum value $T_{\min }$ which depends on the individual open-mindedness $\sigma$, fig. 3 (right). In this range, it is more convenient to wait for the system to begin converging before launching an external propaganda. If one imposes a "too early" propaganda, the individuals with initial opinion close to $O_{p}$ quickly converge together, while the individuals with extreme opposite opinion escape from the clustering and drag a certain number of moderates to form an alternative extremist opposition group. This opposition group has an opinion about the average between the moderate opinion 0.5 and the extreme opposite of propaganda $\left(\left|1-O_{p}\right|\right)$, fig. 4. Indeed, the cluster centered around opinion 0.5 , i.e. the cluster shown by the system in the absence of propaganda, is not a stable solution anymore when a propaganda is imposed. Moderate individuals join one of the two extreme clusters. The propaganda has the effect of extremising the system; indeed, in the presence of propaganda, it always shows one (if $T>T_{\min }$ ) or two (if $T<T_{\min }$ ) opposite extreme clusters. An upper bound estimate of $T_{\min }$ can be derived showing that it scales linearly with $O_{p}$, the slope being proportional to $T_{c}$.

In the case of extreme propaganda (large or small $O_{p}$ ) in a close-minded system (small $\sigma$ ), $100 \%$ efficiency $E_{p}$ may be obtained if the propaganda period $T$ is in between two values $T_{\min }$ and $T_{\max }$ which depend on the value of $\sigma$, fig. 4 . In these ranges, $0.28<\sigma<\left|0.5-O_{p}\right|$ zones III in fig. 2, one must find the optimal balance between two opposite tendencies. On the one hand, it is necessary to wait for the system to naturally converge to a moderate opinion, so that the individuals with opposite extreme opinion with respect to $O_{p}$ come closer to the propaganda. On the other hand, one should prevent the system from letting too many individuals converge to a moderate opinion that falls out of the basin of attraction of the propaganda. Hence $T_{c}$ 

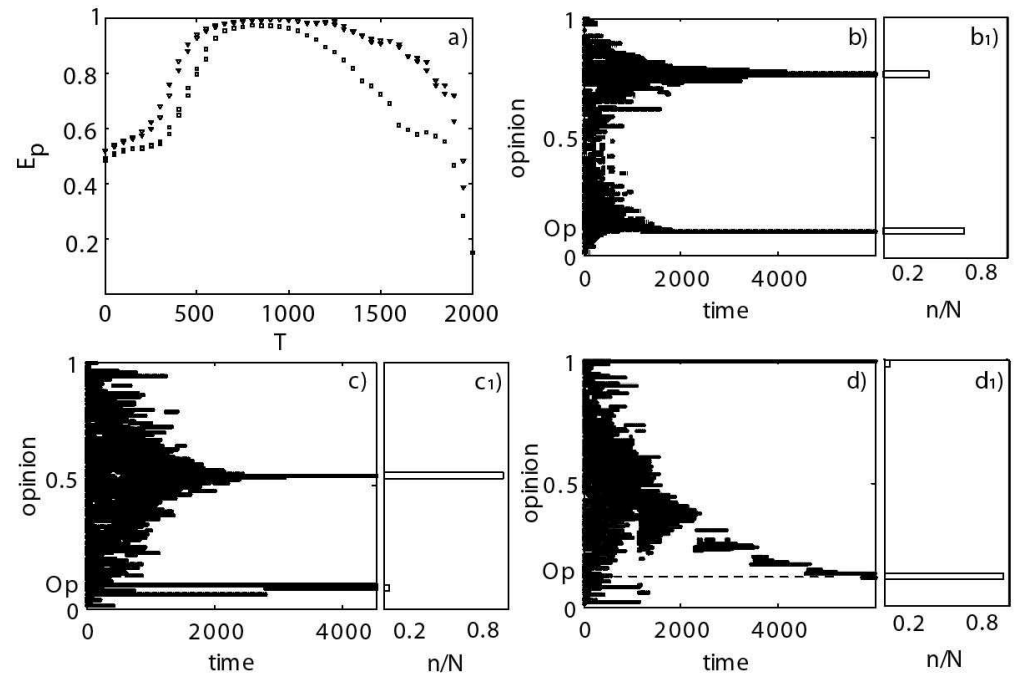

Fig. 4 - Analysis of zones III. In all the subfigures $N=200$ and $O_{p}=0.1$. a) The efficiency $E_{p}$ as a function of the propaganda period $T$ for different values of $\sigma$. The open squares $(\square)$ represent the case $\sigma=0.4$, and the triangles $(\nabla)$ the case $\sigma=0.38$. Note that $T_{\max }$ gets smaller for increased value of $\sigma$. The ansatz $T_{\max } \sim T_{c}$ proposed in the text gets the correct order of magnitude for $T_{\max }$ and the observed dependence on $\sigma$. b) The opinion of the $N$ individuals as a function of time in the case $\sigma=0.4$ and $T=200<T_{\min }$. $\mathrm{b}_{1}$ ) The final (i.e. stable) distribution of the opinion of the $N$ individuals. c) The opinion of the $N$ individuals as a function of time in the case $\sigma=0.4$, and $T=2700>T_{\max } . \mathrm{c}_{1}$ ) The final (i.e. stable) distribution of the opinion of the $N$ individuals. d) The opinion of the $N$ individuals as a function of time in the case $T_{\min }<T=1150<T_{\max }$, and $\sigma=0.4$. $\mathrm{d}_{1}$ ) The final (i.e. stable) distribution of the opinion of the $N$ individuals.

provides a good estimate for $T_{\max }$. It is interesting to notice that if $T<T_{\min }$, as in the zone-II case, the system gives birth to a natural opposition to the propaganda, i.e. a stable cluster at, roughly, the average between the moderate opinion 0.5 and the extreme opposite of propaganda $\left(\left|1-O_{p}\right|\right)$, fig. $4 \mathrm{~b}$. In contrast, if $T>T_{\min }$ the system shows two stable clusters, one at $O_{p}$ and another very close to 0.5. Indeed, there is no extreme opposition created. This is because the efficiency decreases very fast for $T>T_{\max }$. Remarkably, propaganda can obtain $100 \%$ efficiency only with the collaboration of some individuals of the system. Indeed, if $T_{\min }<T<T_{\max }$ the individuals with a moderate initial opinion bring the extremists to a more moderate position, thus making them vulnerable to the propaganda.

In the $\sigma$ range in which the system spontaneously converges to several stable clusters $(\sigma<0.28)$, we find that the efficiency $E_{p}$ has a linear correlation with $\sigma$ and is independent of $O_{p}$ and $T$. In this range, zone IV in fig. 2, there is no difference between radical (close to the extremes 0 and 1) and moderate (close to 0.5 ) opinions and between obsessive (small $T$ ) and non-obsessive (large $T$ ) propaganda. Here, propaganda produces only local effects because it is able to act only on individuals that have an initial opinion close to $O_{p}$. Indeed, the global dynamics of the system is not affected by the propaganda. Only a small variation of a cluster opinion or the merging of two consecutive clusters at very low $\sigma$ can be observed.

Conclusions. - In order to attain the maximum efficiency for a given propaganda $O_{p}$, the optimal range of propaganda period $T$ depends on the characteristic of the system, i.e. the open-mindedness $\sigma$. Besides the case of zone I, which is quite unrealistic because of the overly large open-mindedness $(\sigma>0.5)$, one can focus on the other domains in the $\left(\sigma, O_{p}\right)$-plane 

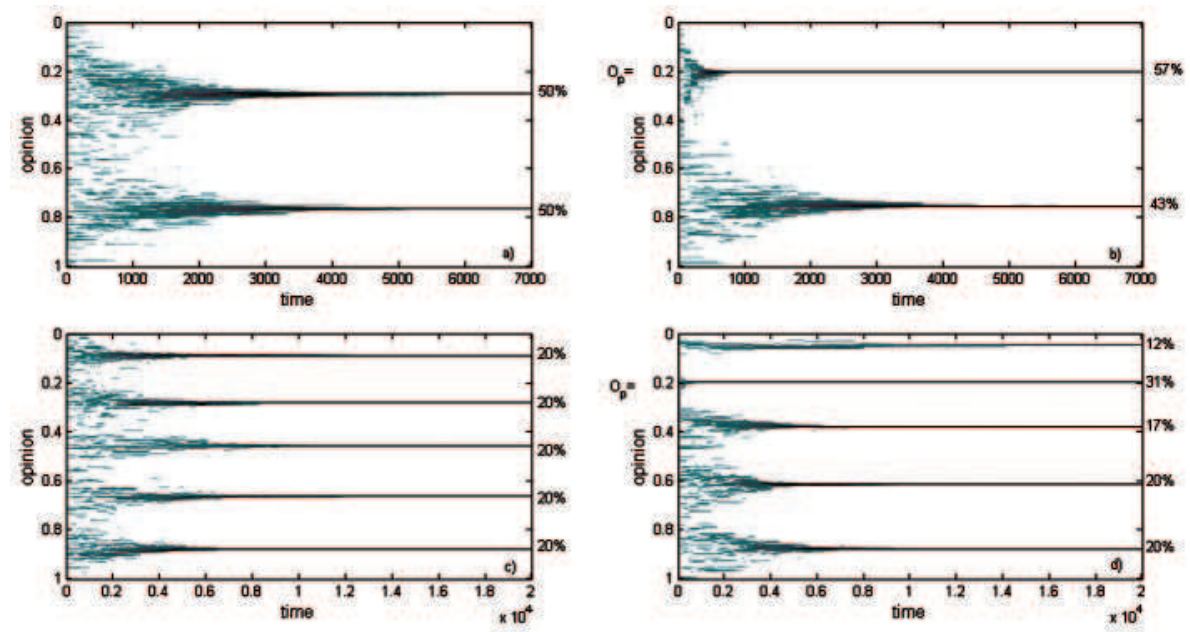

Fig. 5 - Analysis of zones IV. Subfigures show the opinion of the $N=200$ individuals as a function of time. The numbers on the right-hand side of the plot correspond to the fraction of individuals present in the cluster at the end of the simulation. a) Case $\sigma=0.24$, no propaganda is imposed. b) $\sigma=0.24$, $O_{p}=0.2$ and $T=100$. c) $\sigma=0.1$ with no propaganda imposed. d) $\sigma=0.1, O_{p}=0.2$ and $T=100$.

(zones II, III and IV) and relate our mathematical model to actual social systems.

Zones II and III can be compared to a mathematical population that has a single final cluster. In the real world, this social system could mimic a group of people associated to some kind of similar belief: people belonging to the same religion, political party, trade union, etc.

In these $\left(\sigma, O_{p}\right)$ regions, we found that propaganda can have $100 \%$ efficiency if $T>T_{\min }$ and if $T_{\min }<T<T_{\max }$, respectively, for zone II and zones III. This mechanism can also be found in the real-world behaviour. For example, when a leader aims at a moderate redirection of the political line of its own party, he has to act gradually [19-21], i.e. $T>T_{\min }$ as found in zone II.

On the other hand, if a leader wants to give a very extremist (very conservative or progressist) change to the group, the strategy must be set in order to both give time to people to accept the new course and to avoid the formation of consensus of the opposite extreme opinion. This corresponds to the case of zone III, where $T_{\min }<T<T_{\max }$ in order to have $100 \%$ consensus. The above consideration relies on the working assumption that parties initially have uniform distribution of opinions. This ansatz is supposedly correct when new issues arise, which often happens in modern societies, e.g. abortion, immigration, etc.

It is also interesting to notice that in zones II and III, obsessive propaganda $\left(T<T_{\min }\right)$ always gives birth to an opposite extreme cluster. Individuals leave their moderate opinion to join either the extremist opinion or the naturally created opposition. We believe that in the real world this is the well-known over-exposure effect [22]. Public people (or products) do not over-expose themselves in order to prevent an opposite reaction from the audience.

Zone IV of the $\left(\sigma, O_{p}\right)$-plane is maybe the most easily related to a real population. Indeed, $100 \%$ consensus is never reached. In this region one can distinguish between two cases. The case where 2 stable clusters $\left(n_{c}=2\right)$ arise and the one which leads to several organized groups, fig. 5. In the real world this setting could represent, respectively, a country with a bipolar political system (USA, UK) and countries with a multiparty configuration (Italy, France, etc.) [23]. In a multicluster configuration, the model shows that propaganda can, at maximum, create consensus between people with initial opinion close to the propaganda $O_{p}$, 
fig. 5. In the bipolar configuration, our model predicts that people belonging to one side can be reached by an opposite propaganda. This could be interpreted as the fact that in a bipolar system it always happens that some people switch from one side to the other [24], thus making alternance possible. It occurs to many people in the USA to have voted both republican and democratic (or conservative and labor in UK) during their life. It is much less likely to happen that someone votes both a right and a left party in a multiparty country [25]. People usually switch between parties belonging to the same political side.

In conclusion, we showed that the COT opinion model with propaganda is a good candidate as a framework to study real social systems. In particular, it would be interesting to further investigate zone IV of the $\left(\sigma, O_{p}\right)$-plane. It would also be interesting to expand the model by introducing other parameters such as two (or more) competitive propaganda and assigning to the population a more realistic open-mindedness distribution. This would turn the model into a more adapted tool to study real population behaviours.

We thank sociologist L. SCHUFT for her helpful suggestions and enlightening discussions.

\section{REFERENCES}

[1] Hobbes T., The elements of law (1640); The leviathan (1660).

[2] Jones H., Pierre Gassendi (1592-1655): An Intellectual Biography, Nieuwkoop (1981).

[3] Margaret O., Divine Will and the Mechanical Philosophy: Gassendi and Descartes on contingency and necessity in the created world (Cambridge Press) 1994.

[4] Grount's J., Observations on bills of mortality (London) 1662.

[5] Comte A., The Positive Philosophy of Auguste Comte (1896).

[6] Comte A., Système de Politque Positive (1912).

[7] Follmer H., J. Math. Econ., 1 (1974) 51.

[8] Arthur B. W., Increasing Returns and Path Dependence in the Economy (University of Michigan Press, Ann Arbor, MI) 1994.

[9] Galam S., Gefen Y. and Shapir Y., Math. J. Soc., 9 (1982) 1.

[10] Galam S. and Moscovici S., Eur. J. Soc. Psych., 21 (1991) 49.

[11] LATANÉ B. and NowAK A., Self-organizing social systems: Necessary and sufficient conditions for the emergence of clustering, consolidation and continuing diversity, in Progress in Communication Sciences, edited by Barnett G. A. and Boster F. J., 1997, pp. 43-74.

[12] Stone M., Ann. Math. Stat., 32 (1961) 1339.

[13] Chatterjee S. and Seneta E., J. Appl. Prob., 14 (1977) 89.

[14] Cohen J. E., Hajnal J. and Nemman C. M., Stoch. Proc. Appl., 22 (1986) 315.

[15] Laslier J. F., Econ. Appl., 42 (1989) 155.

[16] Krause U., A discrete non-linear and non-autonomous model of consensus formation, in Comm. in Diff. Equ. (Gordon and Breach, Amsterdam) 2000.

[17] Weisbuch G., Deffuant G., Amblard F. and Nadal J. P., Complexity, 7, No. 3 (2002).

[18] Ben-Naim E., Krapivsky P. L. and Redner S., Physica D, 183 (2003) 190.

[19] Machiavelli N., Il Principe (Florence) 1530.

[20] ArgYris C., Increasing Leadership Effectiveness (Wiley, New York) 1976.

[21] Burns J. M., Leadership (Harper Torchbooks, New York, NY) 1978.

[22] Groucutt J., Leadley P. and Forsyth P., Marketing-Essential Principles, New Realities (Kogan) 2004.

[23] CIA, The world factbook 2005, http://www.cia.gov/cia/publications/factbook/.

[24] Flaniganand W. H. and Zingale N., Political Behavior of the American Electorate, 10th edition (Congressional Quarterly Press) 2002.

[25] Pasquino G., La politica italiana. Dizionario critico 1945-95 (Edizioni Laterza) 1995. 